\title{
Profiles of antimicrobial resistance of enterobacteria isolated at livestock enterprises of the Ural region
}

\author{
A. S. Krivonogova ${ }^{1}$, A. G. Isaeva ${ }^{1 \bowtie}$, O. V. Sokolova ${ }^{1}$, K. V. Moiseeva ${ }^{1}$ \\ ${ }^{1}$ Ural Federal Agrarian Research Center of the Ural Branch of the Russian Academy of Sciences, \\ Ekaterinburg, Russia \\ E-mail: isaeva.05@bk.ru
}

Abstract. A study of the antibiotic susceptibility of bacteria of the genus Enterobacter, selected at regional dairy enterprises, was carried out. The purpose of this work was to assess the phenotypic resistance profiles of Enterobacter spp. in the loci of fermenal microbiocenoses related to milk production. Research methodology and methods. In the course of the work carried out, milk, mammary gland secretions, and udder washes from cows at dairy cattle breeding enterprises located in different districts of the Ural region were examined. The phenotypic resistance of Enterobacter spp. Isolates was analyzed to 10 antibacterial drugs: ciprofloxacin, enrofloxacin, ofloxacin, meropenem, doxycycline, chloramphenicol, ceftriaxone, amoxicillin, ampicillin, rifampicin. Results. Average sensitivity values of Enterobacter spp. for all surveyed enterprises were at the level of 2.0-3.3 conventional units (at maximum =4) to target antibiotics, and at the level of 2.0-2.1 conventional units to non-target antibiotics. The highest bactericidal efficacy was found in fluoroquinolones, the lowest in doxycycline and chloramphenicol. For individual enterprises, the average resistance profile included good sensitivity to 3-4 antibiotics, reduced to 4-5 and resistance to 1-2 antibiotics. The main conclusion is that in eight surveyed enterprises, the usual pattern was the resistance of isolates or their low sensitivity to several antibiotics of different classes, which indicates an unfavorable situation with AMR. Scientific novelty. The results obtained in the course of the work performed made it possible to assess the current and actual levels of resistance of Enterobacter spp. Isolates inhabiting those loci of fermenal microbiocenoses that are directly related to milk production.

Keywords: antimicrobial resistance, antibiotic resistance, enterobacteria, Enterobacter spp., Antibiotics, phenotypic antibiotic sensitivity, resistance profile, animal husbandry, milk.

For citation: Krivonogova A. S., Isaeva A. G., Sokolova O. V., Moiseeva K. V. Profiles of antimicrobial resistance of enterobacteria isolated at livestock enterprises of the Ural region // Agrarian Bulletin of the Urals. 2021. No. 08 (211). Pp. 36-41. DOI: 10.32417/1997-4868-2021-211-08-36-41.

Date of paper submission: 01.07.2021, date of review: 09.07.2021, date of acceptance: 12.07.2021.

\section{Introduction}

The fight against antimicrobial resistance (AMR) in animal husbandry is currently not yielding significant results, despite the measures taken. The complexity and diversity of livestock systems, the variability of bacterial communities on farms, the imperfect practice of using antibiotics, their non-therapeutic use as growth and productivity stimulants - all this leads to the circulation of resistant microorganisms, the spread of mobile elements of resistance in the environment and the contamination of meat and dairy products $[1$, p. 1$]$. In addition, accurate diagnostic methods are often unavailable for a livestock enterprise due to the lack of an appropriate laboratory base, and the study of a large number of samples in specialized laboratories is too expensive. As a result, veterinarians have to treat animals empirically to prevent disease from developing. Therefore, the WHO strategy for AMR control in agriculture recommends the use of the Empirical Diagnostic Guidelines, which are based on information on the most relevant diseases in each country, and can help to determine the priority and appropriateness of antibiotic use in different situations. Vaccination of animals, abandonment of antibiotic prophylaxis, improvement of technological conditions for keeping and feeding, increasing the availability of laboratory diagnostics of diseases requiring antibiotic treatment are also of great importance [2, p. 10]. However, in many countries, inappropriate therapeutic tactics and the use of feed additives with an antibacterial component continue to be the main reasons for increasing AMR in livestock and aquaculture. Consequently, livestock continues to be an important source of bacteria containing antimicrobial resistance genes (ARG). To date, the main routes of transmission of resistance agents from animals to humans are known: these are the consumption of products of animal origin contaminated with ARG and the exchange of genetic material between bacteria of livestock and human microbiomes through direct contact within the same ecological niche [3, p. 148], [4, p. 6677]. ARG genes of pathogenic, commensal bacteria, as well as all environmental bacteria, mobile genetic elements and bacteriophages form a single resist, from which opportunistic bacteria of ani- 
Agrarian Bulletin of the Urals No. 08 (211), 2021

mals and humans can acquire resistance [5, p. 173]. At the same time, the distribution of ARG in microbial communities is determined by the phylogeny of bacteria, the coincidence of their ecological niches and the specificity of mobile genetic elements, and horizontal gene transfer (HGT) is associated with the greatest risk of developing resistance [6, p. 30], [4, p. 15], [7, p. 244].

Representatives of the Enterobacter genus play an important role in the development of nosocomial pathologies in humans and opportunistic infections in productive animals. In medicine, two opportunistic pathogens, E. aerogenes and E. cloacae, have recently acquired high clinical significance, while it is noted that blood infections caused by resistant Enterobacter spp. are the most invasive [8, p. 176].

In farm animals, Enterobacter spp. associated with inflammatory processes of the reproductive tract, urinary tract, infections of the udder, skin and soft tissues. And if the cause is multi-resistant bacteria, then antibiotic therapy will be ineffective, and the pathological process becomes protracted or takes on a chronic form. Representatives of the genus Enterobacter spp. with multidrug resistance are often characterized by acquired resistance not only to third generation cephalosporins, but also to carbapenems, which is associated with the plasmid-mediated mechanism of carbapenemase production [9, p. 214], $[8$, p. 176]. The complex mechanisms of resistom functioning at livestock enterprises necessitate constant monitoring and control over the state of the microbial community and its AMR. At the same time, if it is impossible to carry out genetic studies, it seems relevant to screen the phenotypic antibiotic susceptibility of microorganisms.

The purpose of this work was to study the phenotypic antibiotic sensitivity of Enterobacter spp. Isolated from various biotopes of regional dairy enterprises.

\section{Methods}

Milk, mammary gland secretions, udder washings from cows on dairy farms located in different districts of the Sverdlovsk and Chelyabinsk regions were examined. In total, 8 enterprises were examined, 55 samples were taken. Samples were selected at random. The phenotypic resistance of Enterobacter spp. Isolates isolated from samples was analyzed to 10 antibacterial drugs: ciprofloxacin, enrofloxacin, ofloxacin, meropenem, doxycycline, chloramphenicol, ceftriaxone, amoxicillin, ampicillin, rifampicin. Bacteriological studies were carried out in a specialized microbiological laboratory using standard methods in accordance with GOST 32901-2014 "Interstate standard. Milk and dairy products. Methods of microbiological analysis", MUK 4.2.1890-04 "Determination of the sensitivity of microorganisms to antibacterial drugs. Methodical instructions", "Rules for bacteriological study of feed. Approved Main Veterinary Directorate of the Ministry of Agriculture of the USSR June 10, 1975", National Standard GOST R ISO 207761-2010 "Clinical guidelines, approved by at the Expanded meeting of the Interregional Association for Clinical Microbiology and Antimicrobial Chemotherapy (Moscow, 05/15/2017)". The biomaterial from the samples was plated on nutrient media, cultivated, the isolates were identified and tested for sensitivity to antimicrobial drugs
(AMP) by the disk diffusion method $[10,11,12]$. The interpretation of the results was carried out in accordance with the national standard, recommendations and expert rules EUCAST (2018 and 2021). The values of antibiotic sensitivity in the calculations are given in arbitrary units: 0 - resistance, 4 - maximum sensitivity. The results were analyzed using statistical MS Excel methods.

\section{Results}

Studies have shown that at all enterprises, Enterobacter isolates with reduced sensitivity or resistance to target antibiotics were present in the samples (table 1).

Isolates identified at enterprise No. 1 had satisfactory sensitivity to fluoroquinolones and meropenem. At the same time, the sensitivity variability in the sample was relatively low, the most common value was "3 c. u.", which, in our opinion, was a satisfactory indicator. The minimum average values were found in tests with chloramphenicol and doxycycline - an average of 1.7-1.9 c. u. Indicators of antibiotic susceptibility of isolates to nontarget antibiotics (amoxicillin, ampicillin and rifampicin), as expected, were at the level of 1-1.7 a. u., while a significant scatter of results was noted within the tested group of isolates. At enterprise No. 2, a high susceptibility of Enterobacter spp. was revealed to fluoroquinolones, moderate - to chloramphenicol, meropenem, doxycycline. The susceptibility to non-target antibiotics was better than at enterprise No. 1. The results for enterprise No. 3 were generally characterized as good, the average sensitivity of isolates to target antibiotics exceeded $3 \mathrm{c}$. u. with a relatively high homogeneity of indicators. At enterprise No. 4, signs of resistance to chloramphenicol were found: the average level of sensitivity was $0.88 \mathrm{c}$. u., with a standard deviation of the sample of 1.17 - that is, among the investigated isolates, there were both moderately susceptible and completely resistant to this antibiotic, with this resistant prevailed (sample mode $=0$ ). Such a picture may be a consequence of the irrational use of chloramphenicol at the enterprise, when the use of an antibiotic leads to the achievement of therapeutic goals in individual animals, but at the same time contributes to the formation of a pool of resistant isolates throughout the technological area, however, to confirm this conclusion, it is necessary to conduct a study on a larger sample. Analysis of isolates of Enterobacter spp. at enterprise No. 5 revealed similar trends in relation to meropenem, doxycycline and chloramphenicol, however, the level of their sensitivity on average corresponded to I (1.8-2.0 c. u.) and, in accordance with the EUCAST recommendations, can be interpreted as sensitive to high doses of the antibiotic. Isolates at enterprises No. 7 and No. 8 were assessed as sensitive to fluoroquinolones, moderately sensitive to ceftriaxone, doxycycline. The average sensitivity to nontarget antibiotics did not exceed 1.0, with the exception of amoxicillin at enterprise No. 8. The most favorable antibiotic susceptibility profiles of Enterobacter isolates were found at enterprise No. 6 (fig. 1). With the exception of doxycycline (average - 2.0 a. u.) and chloramphenicol (average - 2.6 a. u.), the isolates were well sensitive to all target antibiotics: the average was above 3 values for the sample was insignificant, $\delta$ did not exceed 0.49 , and most often the isolates showed sensitivity at the level of $4 \mathrm{c}$. $\mathrm{u}$. 
Аграрный вестник Урала № 08 (211), 2021 г.

Indicators of antibiotic sensitivity of Enterobacter spp. isolated at eight livestock farms in the Urals region

Ciprofloxacin

Meropenem

Doxycycline

Ofloxacin

Levomycetin

Ceftriaxone

Enrofloxacin

Amoxicillin**

Ampicillin**

Rifampicin**

Antibiotic

Dairy farm No. 1

\begin{tabular}{|c|c|c|}
\hline \multicolumn{3}{|c|}{ Dairy farm No. 1} \\
\hline \multicolumn{3}{|c|}{$(\boldsymbol{n}=\mathbf{1 0})$} \\
\hline Average level & $\boldsymbol{\delta}$ & Mode \\
\hline 3.30 & 0.64 & 3 \\
\hline 3.10 & 0.30 & 3 \\
\hline 1.90 & 0.83 & 2 \\
\hline 3.30 & 0.64 & 3 \\
1.70 & 1.10 & 2 \\
\hline 2.70 & 0.78 & 2 \\
\hline 2.90 & 0.30 & 3 \\
\hline 1.70 & 1.00 & 2 \\
\hline 1.30 & 1.27 & 0 \\
\hline 1.00 & 0.77 & 1 \\
\hline
\end{tabular}

Dairy farm No. 3

\begin{tabular}{|c|c|c|c|c|c|c|}
\hline \multirow{3}{*}{ Antibiotic } & \multirow{2}{*}{\multicolumn{3}{|c|}{$(n=7)$}} & \multirow{2}{*}{\multicolumn{3}{|c|}{$(n=8)$}} \\
\hline & & & & & & \\
\hline & Average level & $\delta$ & Mode & Average level & $\delta$ & Mode \\
\hline Ciprofloxacin & 3.86 & 0.35 & 4 & 3.13 & 0.78 & 3 \\
\hline Meropenem & 3.86 & 0.35 & 4 & 1.50 & 0.87 & 2 \\
\hline Doxycycline & 3.14 & 0.83 & 4 & 1.38 & 0.86 & 2 \\
\hline Ofloxacin & 3.14 & 1.12 & 4 & 3.00 & 0.50 & 3 \\
\hline Levomycetin & 3.43 & 0.49 & 3 & 0.88 & 1.17 & 0 \\
\hline Ceftriaxone & 3.86 & 0.35 & 4 & 3.25 & 0.66 & 3 \\
\hline Enrofloxacin & 3.86 & 0.35 & 4 & 2.75 & 0.43 & 3 \\
\hline Amoxicillin** & 2.43 & 1.40 & 4 & 1.25 & 1.30 & 0 \\
\hline Ampicillin** & 1.57 & 0.90 & 2 & 0.25 & 0.66 & 0 \\
\hline Rifampicin** & 1.14 & 0.83 & 2 & 0.75 & 0.83 & 0 \\
\hline \multirow{3}{*}{ Antibiotic } & \multicolumn{3}{|c|}{ Dairy farm No. 5} & \multicolumn{3}{|c|}{ Dairy farm No. 6} \\
\hline & \multicolumn{3}{|c|}{$(n=5)$} & \multicolumn{3}{|c|}{$(n=5)$} \\
\hline & Average level & $\delta$ & Mode & Average level & $\delta$ & Mode \\
\hline Ciprofloxacin & 3.00 & 0.00 & 3 & 3.60 & 0.49 & 4 \\
\hline Meropenem & 1.80 & 0.75 & 1 & 3.80 & 0.40 & 4 \\
\hline Doxycycline & 2.00 & 0.89 & 1 & 2.00 & 0.63 & 2 \\
\hline Ofloxacin & 2.60 & 0.49 & 3 & 3.80 & 0.4 & 4 \\
\hline Levomycetin & 2.00 & 0.00 & 2 & 2.60 & 0.49 & 3 \\
\hline Ceftriaxone & 2.60 & 0.49 & 3 & 4.00 & 0.00 & 4 \\
\hline Enrofloxacin & 3.00 & 0.00 & 3 & 3.80 & 0.40 & 4 \\
\hline Amoxicillin** & 1.60 & 0.80 & 1 & 3.60 & 0.49 & 4 \\
\hline Ampicillin** & 1.60 & 0.49 & 2 & 1.80 & 0.40 & 2 \\
\hline Rifampicin** & 0.40 & 0.49 & 0 & 1.80 & 0.40 & 2 \\
\hline \multirow{3}{*}{ Antibiotic } & \multicolumn{3}{|c|}{ Dairy farm No. 7} & \multicolumn{3}{|c|}{ Dairy farm No. 8} \\
\hline & \multicolumn{3}{|c|}{$(n=10)$} & \multicolumn{3}{|c|}{$(n=8)$} \\
\hline & Average level & $\delta$ & Mode & Average level & $\delta$ & Mode \\
\hline Ciprofloxacin & 3.33 & 0.47 & 3 & 2.88 & 0.60 & 3 \\
\hline Meropenem & 2.50 & 0.50 & 2 & 1.50 & 0.87 & 2 \\
\hline Doxycycline & 2.17 & 0.37 & 2 & 1.38 & 0.86 & 2 \\
\hline Ofloxacin & 3.33 & 0.47 & 3 & 2.50 & 0.50 & 3 \\
\hline Levomycetin & 3.00 & 0.58 & 3 & 0.88 & 1.17 & 0 \\
\hline Ceftriaxone & 1.17 & 1.07 & 0 & 2.75 & 0.43 & 3 \\
\hline Enrofloxacin & 3.17 & 0.69 & 3 & 2.75 & 0.43 & 3 \\
\hline Amoxicillin** & 0.67 & 0.75 & 0 & 1.25 & 1.30 & 0 \\
\hline Ampicillin** & 0.50 & 0.76 & 0 & 0.25 & 0.66 & 0 \\
\hline Rifampicin** & 0.33 & 0.47 & 0 & 1.00 & 0.71 & 1 \\
\hline
\end{tabular}

* Antibiotic sensitivity values are given in conditional points: 0 - resistance, 4 - maximum sensitivity (sensitivity definition according to [12-14]).

${ }^{*}$ Antibiotic is not targeted against Enterobacter spp. (Natural resistance or unusual phenotype). 
Summary antibiotic sencitivity profiles of Enterobacter spp. isolates from milk samples at farms \#4 and \#6

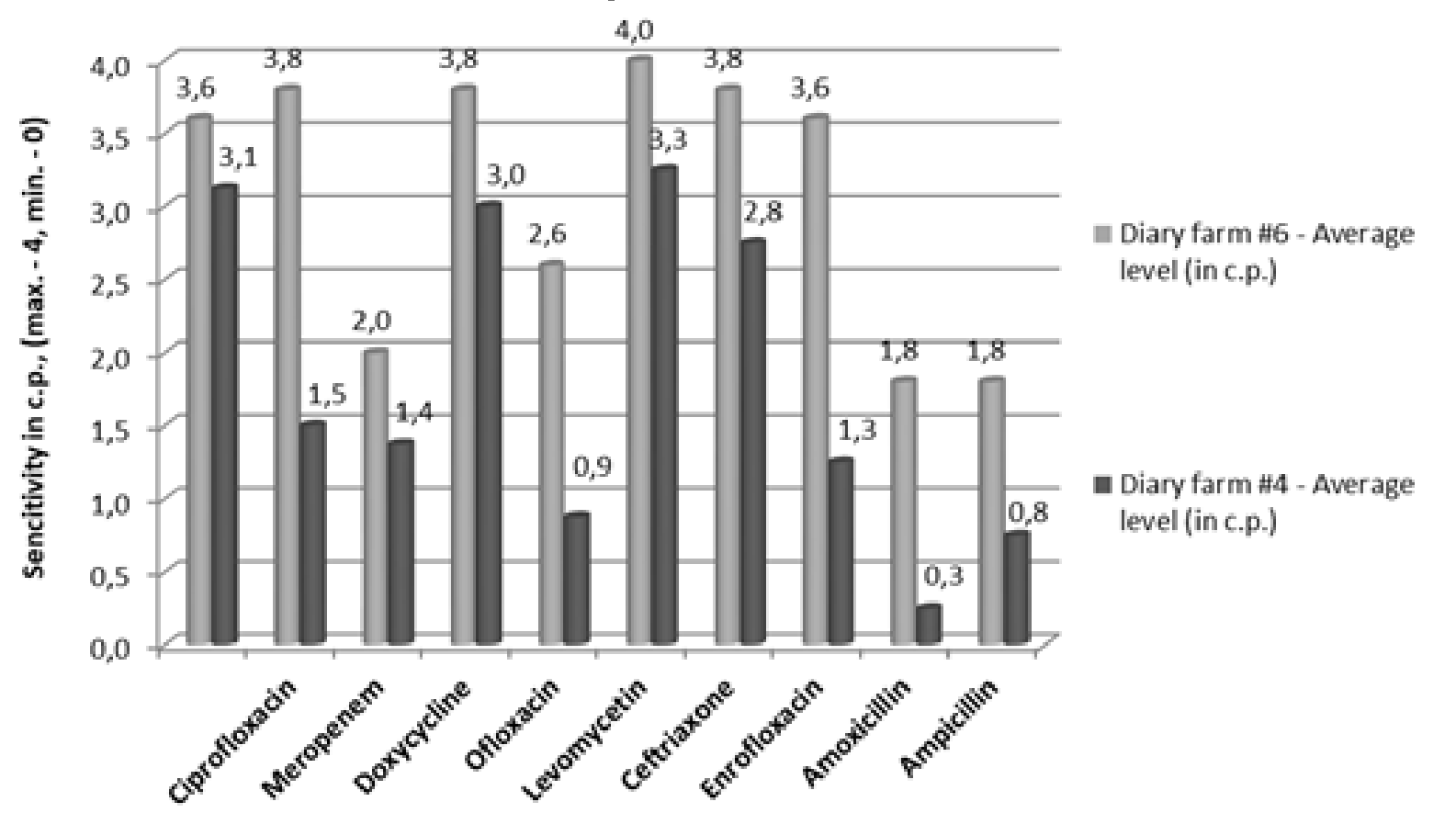

Fig. 1. Summary antibiotic sensitivity profiles of Enterobacter spp. isolates from milk samples at farms No. 4 and No. 6. Values are given in conditional points from 0 to 4

\section{Average sensitivity of Enterobacter spp. isolates to antibiotics at 8 surveyed plants (in c.p., $\max 4$ )}

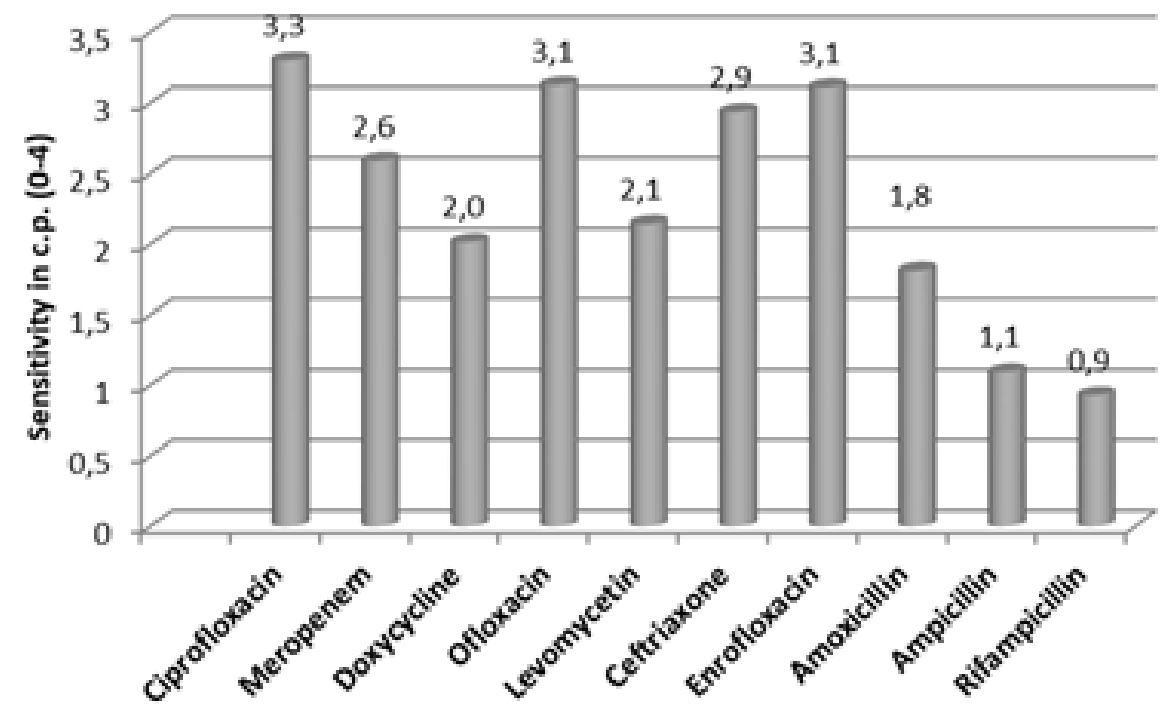

m Average value of antibiotic sensitivity

Fig. 2. The average sensitivity of Enterobacter spp. isolates to antibiotics at 8 surveyed plants. Sensitivity is presented in units, maximum - 4, minimum - 0 , resistance

In general, the average sensitivity of Enterobacter spp. 2.0-2.1 c. u., which corresponds to the intermediate senof all surveyed enterprises to target antibiotics were at the sitivity of the isolates or sensitivity to increased doses of level of 2.0-3.3 conventional units (fig. 2). The best re- the antibiotic. The sensitivity to non-target antibiotics sult was shown by fluoroquinolones (ciprofloxacin, enro- amoxicillin, ampicillin and rifampicin was generally floxacin and ofloxacin), the average sensitivity to which above zero, and, as expected, did not exceed 2.0 c. u., that was assessed as good (above 3 c. u.). The least effective is, it was low. were doxycycline and chloramphenicol - an average of 


\section{Discussion and Conclusion}

The results of this work showed that microbiocenoses at all surveyed enterprises are contaminated with AMR agents, which cause a decrease in the phenotypic sensitivity of individual isolates to antibiotics. This situation could be due to inappropriate antibiotic therapy, the use of antimicrobial drugs as growth promoters, or as inexpensive substitutes for animal hygiene measures to prevent infection in the herd. These factors are currently considered the most frequent and main reason for the spread of AMR in the world [15, p. 1351]. The threshold of the world average amount of antimicrobial drugs per kilogram of animal of $50 \mathrm{mg} / \mathrm{kg}$ adopted as a guideline $[16$, p. 27$]$ is maintained only in certain EU countries, in a number of US states, where it is limited by law. In the Russian Federation, the absence of a ban on feed antibiotics inevitably leads to their widespread use, which results in the appearance of resistance genes in livestock microbiomes. In the course of our studies, we obtained a qualitative confirmation of the circulation of resistant isolates of Enterobacter spp. in loci of microbiomes associated with milk production, which may cause resistance agents to enter milk. In this case, the greatest danger is raw or pasteurized milk, since it retains antibiotic resistance genes, and the mechanism of exchange of resistance factors between $[17$, p. 5] is realized. Further analytical work is needed to better assess the extent of ARG distribution on the surveyed farms. However, attention is drawn to the fact that the number of isolates we found with multiple resistance to different classes of antibiotics was very large. The best antibiotic susceptibility profile of Enterobacter spp. the one where, out of 10 antibiotics, a good sensitivity of isolates to only 6 was found, and to the remaining 4 - moderate sensitivity, and there were no episodes of resistance. The average resistance profile according to our data included good sensitivity to 3-4 antibiotics, reduced to 4-5 and resistance to 1-2 antibiotics. Thus, at eight enterprises, the usual pattern was resistance or low sensitivity to several antibiotics of different classes, which indicates an unfavorable situation with AMR. This fact indicates the danger of ARG contamination of the environment, agrobiocenoses of the enterprise, as well as the possibility of personnel contamination with resistant forms of microorganisms [18, p. 2].

New data presented by Crespo-Piazuelo, et al., Verkola, et al. in 2021, there is a risk of infection with resistant bacteria for people who have direct contact with animals. In particular, Verkola and co-authors studied the prevalence of broad-spectrum beta-lactamases and plasmid beta-lactamases produced by Enterobacteriaceae (ESBL/pAmpC-PE) in Finnish veterinarians and found that $3 \%$ of the examined were carriers of ESBL- and AmpC-producing Escherichia coli [19, p. 3], [20, p. 4]. In this regard, the authors strongly recommend protecting people working in close contact with animals from possible contamination with resistance agents. The results of our studies indicate a high level of AMR on the surveyed farms, which requires taking measures not only to contain resistant strains of microorganisms, but also to protect the personnel working at these facilities.

Acknowledgements

The research was carried out within the framework of the State Assignment of the Ministry of Science and Higher Education of the Russian Federation on topic No. 0532-2021-0004 "Development of methodological approaches to monitoring, control and containment of antibiotic resistance of opportunistic microorganisms in animal husbandry".

\section{References}

1. Li X., Aly S. S., Su Z., Pereira R. V., Williams D. R., et al. Phenotypic Antimicrobial Resistance Profiles of E. coli and Enterococcus from Dairy Cattle in Different Management Units on a Central California Dairy // Journal of Clinical Microbiology. 2018. Vol. 7. Pp. 311-317. DOI: 10.4172/2327-5073.1000311.

2. Global Framework for Development \& Stewardship to Combat Antimicrobial Resistance. Draft Roadmap. WHO/ EMP/IAU/2017.08 (revised 19 October 2017) [e-resource]. URL: https:/www.who.int/antimicrobial-resistance/global-action-plan/UpdatedRoadmap-Global-Framework-for-Development-Stewardship-to-combatAMR_2017_11_03. pdf?ua=1 (date of reference: 11.07.2021).

3. Rodrigues I. A., Ferrari R. G., Panzenhagen P. H. N., Mano S. B., Conte-Junior C. A. Antimicrobial resistance genes in bacteria from animal-based foods // Advances in Applied Microbiology, eds. G. M. Gadd, S. Sariaslani. 2020. Vol. 112. Pp. 143-183. DOI: 10.1016/bs.aambs.2020.03.001.

4. Hu Y., Yang X., Li J., Lu N., Liu F., Wu J., Lin I. Y., Wu N., Weimer B. C., Gao G. F., Liu Y., Zhu B. The Bacterial Mobile Resistome Transfer Network Connecting the Animal and Human Microbiomes // Applied and Environmental Microbiology. 2016. Vol. 82 (22). Pp. 6672-6681. DOI: 10.1128/AEM.01802-16.

5. Von Wintersdorff C. J. H., Penders J., van Niekerk J. M., Mills N. D., Majumder S., van Alphen L. B., Savelkoul P. H. M., Wolffs P. F. G. Dissemination of antimicrobial resistance in microbial ecosystems through horizontal gene transfer // Frontiers in Microbiology. 2016. Vol. 7. Pp. 1-10. DOI:10.3389/fmicb.2016.00173.

6. Ellabaan M. M. H., Munck C., Porse A., et al. Forecasting the dissemination of antibiotic resistance genes across bacterial genomes // Nature Communications. 2021. Vol. 12. Pp. 24-35. DOI: 10.1038/s41467-021-22757-1.

7. Smillie C. S., Smith M. B., Friedman J., Cordero O. X., David L. A., Alm E. J. Ecology drives a global network of gene exchange connecting the human microbiome // Nature. 2011. Vol. 480 (7376). Pp. 241-244. DOI: 10.1038/ nature 10571.

8. Anju V., Siddhardha B., Dyavaiah M. Enterobacter Infections and Antimicrobial Drug Resistance // Model Organisms for Microbial Pathogenesis, Biofilm Formation and Antimicrobial Drug Discovery, eds. B. Siddhardha, et al. 2020. Pp. 175-194. DOI: 10.1007/978-981-15-1695-5_11. 


\section{Agrarian Bulletin of the Urals No. 08 (211), 2021}

9. Liu S., Fang R., Zhang Y., Chen L., Huang N., Yu K., Zhou C., Cao J., Zhou T. Characterization of resistance mechanisms of Enterobacter cloacae Complex co-resistant to carbapenem and colistin // BMC Microbiology. 2021. Vol. 21. Pp. 208-217.

10. Rules for bacteriological examination of fodder. Developed by the All-Union Research Institute of Veterinary Sanitation and specialists of the Main Veterinary Administration of the USSR Ministry of Agriculture. Approved by the Chief Veterinary Administration of the USSR Ministry of Agriculture on June 10, 1975. Ministry of Agriculture of the USSR; Main Veterinary Administration. Moscow: Kolos, 1976. 10 p.

11. Opredelenie chuvstvitel'nosti mikroorganizmov $\mathrm{k}$ antibakterial'nym preparatam (Metodicheskie ukazaniya MUK 4.2.1890-04) [Guidelines for Susceptibility Testing of Microorganisms to Antibacterial Agents (Methodical instructions)] // Clinical Microbiology and Antimicrobial Chemotherapy. 2004. Vol. 6. No. 4. Pp. 306-359. (In Russian.)

12. Determination of the sensitivity of microorganisms to antimicrobial agents. Interpretation and rules for clinical laboratory tests. Version 2018-03 “Clinical Recommendations, approved at the Extended Meeting of the Interregional Association for Clinical Microbiology and Antimicrobial Chemotherapy”. Moscow, 2017. 206 p.

13. The European Committee on Antimicrobial Susceptibility Testing. Breakpoint tables for interpretation of MICs and zone diameters. Version 10.0. European Commission [e-resource], 2020. URL: http://www.eucast.org (date of reference: 07.04.21).

14. ISO 20776-1:2006 "Clinical laboratory testing and in vitro diagnostic test systems - Susceptibility testing of infectious agents and evaluation of performance of antimicrobial susceptibility test devices. Part 1: Reference method for testing the in vitro activity of antimicrobial agents against rapidly growing aerobic bacteria involved in infectious diseases (IDT). Moscow: Standartinform, 2011. 14 p.

15. Van Boeckel T. P., Glennon E. E., Chen D., Gilbert M., Robinson T. P., Grenfell B. T., Levin S. A., Bonhoeffer S., Laxminarayan R. Reducing antimicrobial use in food animals // Science. 2017. Vol. 357 (6358). Pp. 1350-1352. DOI: $10.1126 /$ science.

16. O'Neill J. Tackling drug-resistant infections globally: Final report and recommendations (Review on Antimicrobial Resistance, 2016) [e-resource] // HM government UK. 2016. URL: https://amr-review.org/Publications.html. (date of reference: 07.04.21).

17. Liu J., Zhu Y., Jay-Russell M., Lemay D., Mills D. Reservoirs of antimicrobial resistance genes in retail raw milk // Microbiome. 2020. Vol. 8 (1). Pp. 1-15. DOI: 10.1186/s40168-020-00861-6.

18. Mitchell S., Bull M., Muscatello G., Chapman B., Coleman N. The equine hindgut as a reservoir of mobile genetic elements and antimicrobial resistance genes // Critical reviews in microbiology. 2021. Vol. 47. Pp. 1-20. DOI: 10.1080/1040841X.2021.1907301.

19. Crespo-Piazuelo D., Lawlor P. Livestock-associated methicillin-resistant Staphylococcus aureus (LA-MRSA) prevalence in humans in close contact with animals and measures to reduce on-farm colonization // Irish Veterinary Journal. 2021. Vol. 74. Pp. 1-12. DOI: 10.1186/s13620-021-00200-7.

20. Verkola M., Pietola E., Järvinen A., Lindqvist K., Kinnunen P., Heikinheimo A. Low prevalence of zoonotic multidrug-resistant bacteria in veterinarians in a country with prudent use of antimicrobials in animals // Zoonoses and public health. 2019. Vol. 66. Pp. 667-678. DOI: 10.1111/zph.12619.

\section{Authors'information:}

Anna S. Krivonogova ${ }^{1}$, doctor of biological sciences, leading scientific researcher, ORCID 0000-0003-1918-3030, AuthorID 683239; +7 (343) 257-20-44

Albina G. Isaeva1, doctor of biological sciences, leading scientific researcher, ORCID 0000-0001-8395-124, AuthorID 665717; +7 (343) 257-20-44

Olga V. Sokolova ${ }^{1}$, candidate of biology sciences, senior scientific researcher, ORCID 0000-0002-1169-4090, AuthorID 648613; +7 (343) 257-20-44

Ksenia V. Moiseeva1 ${ }^{1}$, junior scientific researcher of laboratory, ORCID 0000-0002-9858-1880, AuthorID 779572; +7 (343) 257-20-44

${ }^{1}$ Ural Federal Agrarian Research Center of the Ural Branch of the Russian Academy of Sciences, Ekaterinburg, Russia 